Research Paper

\title{
Simultaneous Inhibition of Cell-Cycle, Proliferation, Survival, Metastatic Pathways and Induction of Apoptosis in Breast Cancer Cells by a Phytochemical Super-Cocktail: Genes That Underpin Its Mode of Action
}

Allal Ouhtit ${ }^{1,2^{*}}$, Rajiv Lochan Gaur ${ }^{1,3^{*}}$, Mohamed Abdraboh ${ }^{1,4^{*}}$, Shubha K. Ireland ${ }^{*}$, Prakash N Rao ${ }^{6}$, Shailaja G Raj7, Hamad Al-Riyami², Somya Shanmuganathan², Ishita Gupta², Subramanyam N Murthy ${ }^{8}$, Andrew Hollenbach ${ }^{9}$, and Madhwa HG Raj ${ }^{1,10}$

1. Stanley S Scott Cancer Center, Louisiana Health Sciences Center, New Orleans, Louisiana

2. Present address: Department of Genetics, College of Medicine and Health Sciences, Sultan Qaboos University, Oman;

3. Present address: Department of Pathology, Stanford University, California.

4. Present address: Faculty of Science, University of Mansora, Egypt

5. Department of Biology, Xavier University of Louisiana, New Orleans, Louisiana.

6. New Jersey Organ and Tissue Sharing Network, New Jersey.

7. Protegene Corporation, Metairie, Louisiana.

8. Departnent of Environmental Toxicology, Southern University and A \& M College, Baton Rouge, Louisiana,

9. Department of Genetics, LSU Health Sciences Center, New Orleans, Louisiana, USA,

10. Department of Obstetrics \& Gynecology, Louisiana Health Sciences Center.

* These four authors equally contributed to this work.

$\triangle$ Corresponding author: Madhwa H.G. Raj, Ph.D, Professor, Department of Ob-Gyn \& Stanley S Scott Cancer Center, LSU Health Sciences Center. 1542 Tulane Ave, New Orleans, LA 70112. Ph: (504) 296-2570. mraj@lsuhsc.edu ; rajmadhwa@yahoo.com

(c) Ivyspring International Publisher. This is an open-access article distributed under the terms of the Creative Commons License (http://creativecommons.org/ licenses/by-nc-nd/3.0/). Reproduction is permitted for personal, noncommercial use, provided that the article is in whole, unmodified, and properly cited.

Received: 2013.07.23; Accepted: 2013.08.17; Published: 2013.II.14

\begin{abstract}
Traditional chemotherapy and radiotherapy for cancer treatment face serious challenges such as drug resistance and toxic side effects. Complementary / Alternative medicine is increasingly being practiced worldwide due to its safety beneficial therapeutic effects. We hypothesized that a super combination (SC) of known phytochemicals used at bioavailable levels could induce $100 \%$ killing of breast cancer (BC) cells without toxic effects on normal cells and that microarray analysis would identify potential genes for targeted therapy of BC. Mesenchymal Stems cells (MSC, control) and two $B C$ cell lines were treated with six well established pro-apoptotic phytochemicals individually and in combination (super cocktail), at bioavailable levels. The compounds were ineffective individually. In combination, they significantly suppressed $B C$ cell proliferation (>80\%), inhibited migration and invasion, caused cell cycle arrest and induced apoptosis resulting in $100 \%$ cell death. However, there were no deleterious effects on MSC cells used as control. Furthermore, the SC down-regulated the expression of PCNA, Rb, CDK4, BcL-2, SVV, and CD44 (metastasis inducing stem cell factor) in the $B C$ cell lines. Microarray analysis revealed several differentially expressed key genes (PCNA, Rb, CDK4, Bcl-2, SVV, P53 and CD44) underpinning SC-promoted BC cell death and motility. Four unique genes were highly up-regulated (ARC, GADD45B, MYLIP and CDKNIC). This investigation indicates the potential for development of a highly effective phytochemical combination for breast cancer chemoprevention / chemotherapy. The novel over-expressed genes hold the potential for development as markers to follow efficacy of therapy.
\end{abstract}

Key words: Breast cancer; phytochemicals; chemoprevention; microarray; metastasis 


\section{INTRODUCTION}

Cancer is the second leading cause of mortality in the U.S with most of the deaths resulting from metastatic tumor formation at secondary sites. Deaths occur despite radio- and/or chemotherapy treatments [1]. One of the primary causes of this high rate of tumor recurrence and mortality is due to a small population of cancer stem cells (CSC) that evade therapy. The stem cells are characterized by their ability to generate new tumors and their frequent multi-drug resistance (MDR) [2]. Therefore, it is critical to develop new chemotherapeutic drugs or alternative approaches to treatment that are safer for the patient and more effective in eradicating the tumor One such alternative is the use of naturally occurring phytochemicals present in foods such as vegetables, fruits, spices and plant roots [3]. Recent reports have demonstrated anti-oxidant, anti-inflammatory, antiproliferative and pro-apoptotic effects of various phytochemicals [3-5] Moreover, the pro-apoptotic and anti-proliferative effects of phytochemicals indicate their ability to inhibit the growth of several types of cancers of blood, skin, brain, colon, ovaries, breast, prostate and pancreas [2]. However, existing data using either individual and/or combination of 2 to 3 phytochemicals in in-vitro and in-vivo cancer models did not demonstrate a complete eradication of cancer cells [6-8].

Several studies have been conducted to elucidate the mode of action of a number of phytochemicals. The anti-cancer effect of Curcumin (Curcuma root extract, also known as turmeric) results from its ability to inhibit tumor growth and metastasis. Curcumin and its derivatives inhibit the proliferation of breast cancer (BC) cell lines and induce apoptosis [9-11]. In the $\mathrm{BC}$ cell line MDA-MB-231, cellular proliferation was inhibited via down-regulation of the expression of the cell cycle regulator cyclin D and NF-KB. Further, metastasis was inhibited through down-regulation of the expression of MMP-1[12].

Isoflavone (Genistein), a naturally occurring chemical in soybeans, has a protective effect against localized prostate cancer, non-small cell lung cancer, and estrogen and progesterone receptor positive $(\mathrm{ER}+, \mathrm{PR}+)$ breast tumors [6,13-15]. Using similar mechanisms to that of Curcumin, Genistein sensitizes cancer cells to chemotherapeutic drugs and induces breast, pancreatic and prostate cancer cell death by promoting the expression of pro-apoptotic proteins, inactivating NF-KB, and inducing cell cycle arrest [16-18].

Indol-3-Carbinol (I3C), extracted from cruciferous plants, plays an important role in inhibiting carcinogenesis by protecting cells from oxidative stress due to formation of reactive oxygen species (ROS), known to promote cancer development [19]. The chemical derivative of I3C, 1-Benzyl-indole-3-carbinol has a 1000 fold higher activity than I3C in inhibiting the growth of both estrogen-dependent and -independent breast tumors [20]. I3C also plays an important role in sensitizing $\mathrm{BC}$ cells to the chemotherapeutic drug tamoxifen [20]. In MDA-MB-231 BC cell line, another member of I3C, 3'-diindolylmethane (DIM) induced apoptosis and inhibited angiogenesis by suppressing the activity of the Akt/NF-KB signaling pathway. I3C was shown to inhibit bone metastasis of MDA-MB-231 breast cancer cells in a SCID mouse model [21].

In a recent study, extract from the blue green algae Spirulina platensis, combined with selenium (an element with anti-cancer activity), was shown to inhibit the growth of MCF7 BC cell line. This combination is believed to induce cell cycle arrest at G1 stage by inhibiting cyclin dependent kinases CDK4 and CDK6 and their partners cyclin D1 and cyclin D3. Spirulina extracts also increased the level of the tumor suppressor p53 and p21Cip1/WAF1 and triggered DNA fragmentation, up-regulated the expression of the pro-apoptotic proteins Bax, Caspase-8, Caspase-9, and the cleavage of DNA repairing enzyme poly (ADP) ribose polymerase (PARP) [22]. The active compound of these extracts, C-phycocyanin (C-PC) is a water-soluble biliprotein that has anti-inflammatory and anti-oxidant effects and has been reported to induce apoptosis in MCF7 breast cancer cells [22]. Our previous studies have demonstrated that spirulina inhibited rat liver toxicity and carcinogenesis induced by dibutyl nitrosamine (DMB) precursors [23]. We showed inhibition of $\mathrm{Bcl} 2$ and $\mathrm{RB}$ expression as well as increased P21 and Bax during this chemoprevention.

Grape seed extract contains Resveratrol (RE) that inhibits cancer cell proliferation by triggering cell cycle arrest through cell cycle regulatory proteins such as cyclin E and cyclin D1. Furthermore, resveratrol induces apoptosis by up-regulating the expression of tumor suppressor genes p21Cip1/WAF1, p53, the pro-apoptotic protein Bax, activating Caspase apoptotic signals, and down-regulating the expression of the anti-apoptotic proteins $\mathrm{Bcl}-2, \mathrm{Bcl}-\mathrm{X}_{\mathrm{L}}$ and survivin [24-26] We demonstrated that resveratrol synergizes with Indole 3 Carbinol to inhibit proliferation and survival of ovarian cancer cells, by down regulating SVV [27].

Quercetin is a plant-derived flavonoid present in fruits, vegetables and tea [28]. Quercetin induces cell apoptosis through a multi-targeting mechanism by inducing the expression of Bax and activating TRAIL-induced apoptosis. Quercetin also suppresses the activity of Bcl-2 protein family and induces the 
DNA fragmentation process [28-30].

In addition to the mechanisms described above, phytochemicals can also exert anti-metastatic action by altering the activity and/or expression of some cell adhesion molecules that are mainly responsible for cancer promotion $[31,32]$. One such molecule, CD44, is significantly up-regulated during cancer cell growth, primarily during metastasis. In addition, CD44 is responsible for cell motility and contributes to the ability of cells to metastasize [33].

The aim of the present investigation is to analyze the ability of combinations of the naturally available phytochemicals to inhibit cancer cell growth migration and invasion, and induce apoptosis, when used at bioavailable levels. Use of phytochemicals may provide a promising strategy for treating cancer without harmful side effects that are usually observed in the currently used chemo- and radio-therapies.

\section{MATERIALS AND METHODS}

\section{Cell culture and proliferation assay in the presence of Phytochemicals}

MDA-MB-231 and MCF-7 BC cell lines were plated on clear bottom black 96 well plates (2000 cells/well) and cultured in DMEM supplemented with $10 \%$ fetal bovine serum (FBS) and $1 \%$ penicillin and streptomycin (0.1 ml/well). Mesenchymal Stem Cells (MSCs), kindly provided by Dr. David Welsh (LSU Health Sciences Center, New Orleans), were cultured in MEM-Alpha (GIBCO, Gaithersburg, MD) supplemented with $16.5 \%$ of Bovine Serum Albumin (BSA) (Atlanta Biologicals, USA) and 1\% L-Glutamine (GIBCO, Gaithersburg, MD). The phytochemicals Indol-3-Carbinol (I3C), Resveratrol (RE), C-phycocyanin (PC), Isoflavone (Genistein, GA), Curcumin (CUR) and Quercetin (Qurc) (Sigma, St. Louis, MO) were dissolved in $70 \%$ ethanol and used at bioavailable levels (I3C: $4 \mu \mathrm{g} / \mathrm{ml}$; RE: $0.5 \mu \mathrm{g} / \mathrm{ml}$; GA: $3 \mu \mathrm{g} / \mathrm{ml}$; CUR: $2.25 \mu \mathrm{g} / \mathrm{ml}$; PC: $50 \mu \mathrm{g} / \mathrm{ml}$ and Qurc: $1.5 \mu \mathrm{g} / \mathrm{ml}$ ). The concentration of PC $(50 \mu \mathrm{g} / \mathrm{ml})$ was determined after titration with a range of concentrations (1-500 $\mu \mathrm{g} / \mathrm{ml})$. For treatment of cells, stocks were prepared from each of the phytochemicals in a way that a single well received not more than $10 \mu \mathrm{l}$ of ethanol. Control wells received $10 \mu \mathrm{l}$ of ethanol (vehicle), a nontoxic dose of alcohol as determined from previous studies. The suppression of cell growth was determined using the Alamar Blue cell proliferation assay (Alamar Biosciences, Sacramento, CA), according to the manufacturer's specifications. The oxidized form of this dye, which is non-toxic to cells, is converted to the reduced form by mitochondrial enzyme activity of the viable cells. The shift in fluorescence was measured at $570 \mathrm{~nm}$ (excitation) and $600 \mathrm{~nm}$ (emission) in a Fluo- rometer (LabSystems Fluoreskan-II) $4 \mathrm{~h}$ after addition of the dye. The results are expressed as a percent of MSCs proliferation. MSCs were used in all experiments as negative controls.

\section{Wound healing assay}

BC cell lines MCF7 and MDA-MB-231 were seeded in six-well plates $\left(5 \times 10^{5}\right.$ cells/well) and allowed to adhere for $24 \mathrm{~h}$. The cells were kept at $2 \%$ FBS overnight for synchronization. The cells were washed with phosphate buffer saline (PBS), scratched with a pipette tip in the middle of the plate, and was then washed with PBS to remove the cells which had detached during the scratch [34]. After washing with PBS, media was added containing various concentrations of the phytochemicals, either individually or in combination and photographed at at 5,10,16 and 31 hrs.

\section{Invasion assay}

MCF7 and MDA-MB-231 cells were cultured on $60 \mathrm{~mm}$ dishes and allowed to adhere for $24 \mathrm{~h}$, washed with (1X) sterile phosphate buffer saline (PBS) and replaced with fresh media containing the six phytochemicals as stated above. After $24 \mathrm{~h}$, cells were washed twice with PBS, trypsinized and collected. The harvested cells (50,000cells/well) were re-suspended in DMEM supplemented with 0.5\% BSA. MCF7 and MDA-MB-231 cells were respectively plated in $12 \mu \mathrm{m}$ and $8 \mu \mathrm{m}$ pore size Millicell culture inserts (Millipore, MA) previously coated with a thin layer of $200 \mu \mathrm{g} / \mathrm{ml}$ of Matrigel ${ }^{\mathrm{TM}}$ (BD Biosciences, MA). The inserts containing the cells were placed into a tissue culture dish (lower chamber) with the "attracting medium" which consisted of DMEM supplemented with $10 \%$ FBS. Cells were incubated for $22 \mathrm{~h}$ at $37^{\circ} \mathrm{C}$ after which the Millicell culture insert was removed and the upper surface of the insert was wiped with a cotton swab to remove non-invasive cells. The inserts were dried under laminar flow hood, the cells present on the bottom of the filter (the invasive cells) were stained using the Diff-Quick staining kit, according to the manufacturers' protocol (Dade Behring Inc., Illinois, USA), and the stained cells were counted under a phase-contrast microscope equipped with ocular grids.

\section{Cell cycle analysis}

MCF7 and MDA-MB-231 cells were plated in 60 $\mathrm{mm}$ dishes $\left(1 \times 10^{6} \mathrm{cells} / \mathrm{dish}\right)$ and were allowed to attach for $24 \mathrm{~h}$. The cells were synchronized by culturing them overnight in media with $1 \%$ FBS. The cells were washed twice with PBS and the culturing media was replaced with fresh media enriched with 10\% FBS along with the super combination of the six phytochemicals. At time points of $0,6,12,24 \mathrm{~h}$ the cells were 
harvested by trypsinization, washed with PBS, fixed overnight in $70 \%$ ice cold ethanol, and the DNA was stained with propidium iodide after RNAse treatment according to standard protocol [27,]. Cell cycle analysis was performed by flow-cytometry (BD Biosciences, USA) and cells in G0/G1, S, G2/M and the sub-G0/G1 (apoptotic) phases were quantified.

\section{Western blot analysis}

We analyzed the expression levels of proteins involved in the molecular pathways such as cell cycle regulation and apoptosis by Western blot analysis. MDA-MB-231 and MCF-7 cells were treated with the same concentrations of the above-described phytochemicals. Following 24 and 48 hours of treatment, total cell lysates were collected using RIPA buffer (Santa Cruz, CA). Equal amounts $(30 \mu \mathrm{g})$ of total cell extracts were separated by $12 \%$ SDS-PAGE, transferred to nitrocellulose membranes, and Western blot analysis was performed by probing the membranes with a number of primary antibodies as follows: anti-rabbit Bcl-2 (1:500 dilution; BD Pharmingen), anti-mouse PCNA (1:500 dilution; DAKO), anti-mouse CD44 (1:1000 dilution; R\&D Biosystems), anti-rabbit CDK-4, SVV, p53, and Rb (1:500 dilution, Santa Cruz Biotechnology, CA). Proteins were visualized using Supersignal horseradish peroxidase according to the manufacturer's instructions (West Femto Super Signal, Thermo scientific). Equal loading of the protein samples was assessed by re-probing the membrane with Actin antibody (1:2000 dilution; Santa Cruz Biotechnology, CA).

\section{Microarray Analysis}

MDA-MB-231 cells were treated with ethanol alone (control) or with the above-mentioned concentrations of the six phytochemicals for 6, 12 and $24 \mathrm{~h}$. Procedures for cDNA synthesis, labeling, and hybridization were carried out as described by the manufacturer (Affymetrix, Redwood City, CA). All experiments were performed using human genome U133 plus 2.0 GeneChips as described by the manufacturer. Total RNA was extracted using the Qiagen RNeasy kit according to the manufacturer's protocol. The quantity and quality of the RNA were analyzed using the NanoDrop ND-1000 Spectrophotometer and RNA Nanochip and Bioanalyzer 2100 (Agilent, USA). All RNA samples exhibited a RIN value of 7 or greater. Briefly, $100 \mathrm{ng}$ of total RNA was used for first-and second strand synthesis and in vitro transcription (IVT) reaction using the 3 '-IVT Express Kit. The mRNA was processed for hybridization using the GeneChip Hybridization, Wash, and Stain Kit. For overnight hybridization, $15 \mu \mathrm{g}$ of fragmented mRNA was used in the Hybridization Oven 640, washed, stained with streptavidin-phycoerythrin using a microfluidics workstation, and scanned with the High Resolution 3000 7G Scanner (Affymetrix). Signal and background intensities were quantified by pixel intensity, and expression signals were analyzed using the GeneChip Operating Software (GCOS 1.4). Array quality assessment was analyzed using GeneChip Expression Console. In brief, the raw CEL files were processed in Expression Console using the robust multichip average (RMA) workflow for $3^{\prime}$-expression arrays. All array images and quality control measurements were consistent and within acceptable limits.

The RMA normalized log2-data file from each array was exported and analyzed for fold change relative to the control sample. In addition, detection of call metrics (present, marginal, absent) were determined using the MAS5 algorithm (Affymetrix). Transcripts that were absent across all conditions were removed from further analysis and the top 100 transcripts with the largest fold change were used for further analysis.

To assess the integrity and specificity of the Affymetrix probe sets to detect a single unique transcript, analysis was carried out using the GeneAnnot application (http://genecards.weizmann.ac.il/ geneannot/index.shtml). Probe sets that recognized multiple genes or transcripts were removed. The high integrity transcripts were used for functional analysis using the DAVID Bioinformatics Resource 6.7 (http://david.abcc.ncifcrf.gov/). Enrichment analysis for pathways and gene ontology was carried out using default setting.

\section{Statistical analysis}

Data from triplicates were analyzed by Prizm software and Means were calculated and compared using t- test. Results were presented as Mean \pm S.E.M. of at least triplicates or replicates from three experiments and the data were analyzed statistically using Newman-Keuls multiple comparison test and Student's t-test using Graph Pad Prism 2.01. Differences with $\mathrm{P}<0.05$ were considered significant.

\section{RESULTS}

\section{Assessment of the effects of phytochemicals on cell proliferation}

We were interested in determining the effects of phytochemicals on BC cell lines (MCF7 and MDA-MB-231) when used in combination at physiologically-relevant doses achievable with oral dosing (bioavailable levels). Pursuant to this goal, we tried several combinations of ten phytochemicals and analyzed their ability, both individually and in combina- 
tion, to inhibit cell proliferation and/or induce cell death (data not shown). Based on this preliminary analysis a combination of six phytochemicals (6-combination) was identified. In order to 'determine the effect of these six phytochemicals on cellular proliferation, Alamar-Blue assay was used as an indicator for the number of viable cells. In this assay, treatment of MSCs with the 6-combination was used as a control and was considered as $100 \%$ in comparison with BC cells treated with the various phytochemicals. A moderate effect was observed for each of I3C and Quercetin on MCF7 cell proliferation by day five and six of individual phytochemical treatment (fig.1, left panel). In contrast to the results observed for the individual compounds, a moderate, yet significant decrease of cellular proliferation was observed in the presence of the combination treatment on the first day of treatment. This effect continued throughout the treatment reaching maximum level of inhibition by day six with an 8-fold decrease of cell proliferation (Figure 1). Similarly, the treatment of MDA-MB-231 metastatic BC cell line with each phytochemical individually resulted in no detectable effect on the proliferation of the treated cells throughout the course of the experiment, with the exception of a moderate inhibition with I3C by the third day of treatment. Similar to what was observed with the MCF7 cells, treatment with the 6-combination on the MDA-MB-231 cells showed a significant suppression in the number of proliferating cells by day six (Figure 1, right panel). When the cells were exposed to the phytochemical combination for 8 days, all the cells were found to detach, float up and were lost. These data suggest a synergistic mode of action of the individual phytochemicals in the 6-combination treatment for affecting the proliferation of $\mathrm{BC}$ cells.

In order to determine whether cell death contributed to these observed effects on proliferation, we examined the cellular morphology of MCF7 and MDA-MB-231 BC cells on day 1 and day 2 of 6-combination treatment using phase contrast microscopy. MCF7 and MDA-MB-231 cells exhibited a smooth epithelial cell pattern with prominent nuclei on day 0 of experiment (before treatment). In contrast both MCF7 and MDA-MB-231 cells treated with the 6-combination started to lose cell-cell contact after 24 $\mathrm{h}$. After $48 \mathrm{~h}$ the cells detached from the surface of the tissue culture dish, indicating the cell death (Figure 2).

In contrast, cells treated with the vehicle control ( $1 \%$ ethanol) showed no detectable effects on the cultured cells at both day 1 and day 2 of treatment (data not shown). These results indicate that the observed decrease in proliferation rate may be the result of reduced cell numbers due to increased cell death.
Effect of the six Phytochemicals combination on apoptosis of MCF7 and MDA-MB-23 I cell lines

In order to determine whether the observed inhibition of cell proliferation was caused by an increase in apoptosis, a cell cycle FACS analysis was conducted on MCF7 and MDA-MB-231 cells treated with the 6-combination for different time intervals $(0,6,12$ and $24 \mathrm{~h}$ ). We determined the amount of sub-2N DNA species, which is indicative of DNA fragmentation characteristic of apoptosis. Treatment of both cell lines with the 6-combination resulted in a significant increase of sub-2N DNA species, indicative of the induction of apoptosis reaching $40-50 \%$ for MCF7 cells and only $5 \%$ for MDA-MB-231 cells by $24 \mathrm{~h}$ of treatment (Figure 3). The differences in the extent of apoptosis between the two cell lines is consistent with our proliferation data (Figure 1), which may result from an increased resistance of metastatic BC cell lines to the phytochemical treatment.

\section{Effect of SC treatment on cell migration and invasion}

Treatment of MCF7 cells with the phytochemical combination inhibited their migratory ability, represented by the inability of these cells to close the wound after $31 \mathrm{~h}$ of treatment (figure not shown), a time period in which the control cells could completely heal the wounded area. Moreover, the highly metastatic MDA-MB-231 cells showed similar results to that of MCF7 cells. The untreated cells closed the wounded area by $10 \mathrm{~h}$ in comparison to the 6-combination treated cells that took more than $16 \mathrm{~h}$ to close the same area (figure not shown).

In the invasion assay we observed a greater than $80 \%$ reduction in cell invasion ability upon treatment with the 6-combination therapy, a result observed with both MCF7 and MDA-MB-231 cells (Figure 4).

\section{Mechanism of action of the six phytochemicals in combination}

In western blot analysis of both cell lines we observed a significant decrease in expression of the cell proliferation marker PCNA, the cell cycle regulator $\mathrm{Rb}$ and the cell cycle-dependent kinase CDK4 (Figure 5 , top panel). We also observed a significant decrease in the expression of the anti-apoptotic proteins Bcl-2, SVV and the cell motility protein CD44 Interestingly, we observed a significant decrease in the expression of mutated p53, the oncogenic form of p53, in the MDA-MB-231 cells. In contrast, we observed a highly significant (29 fold) induction of the wild-type p53, the tumor suppressor form of p53 in the MCF7 cells (Figure 5, bottom panel). Taken together, these data support our hypothesis that the 6-combination ther- 
apy plays a central role in the induction of cancer cell death through simultaneous targeting of several dif-
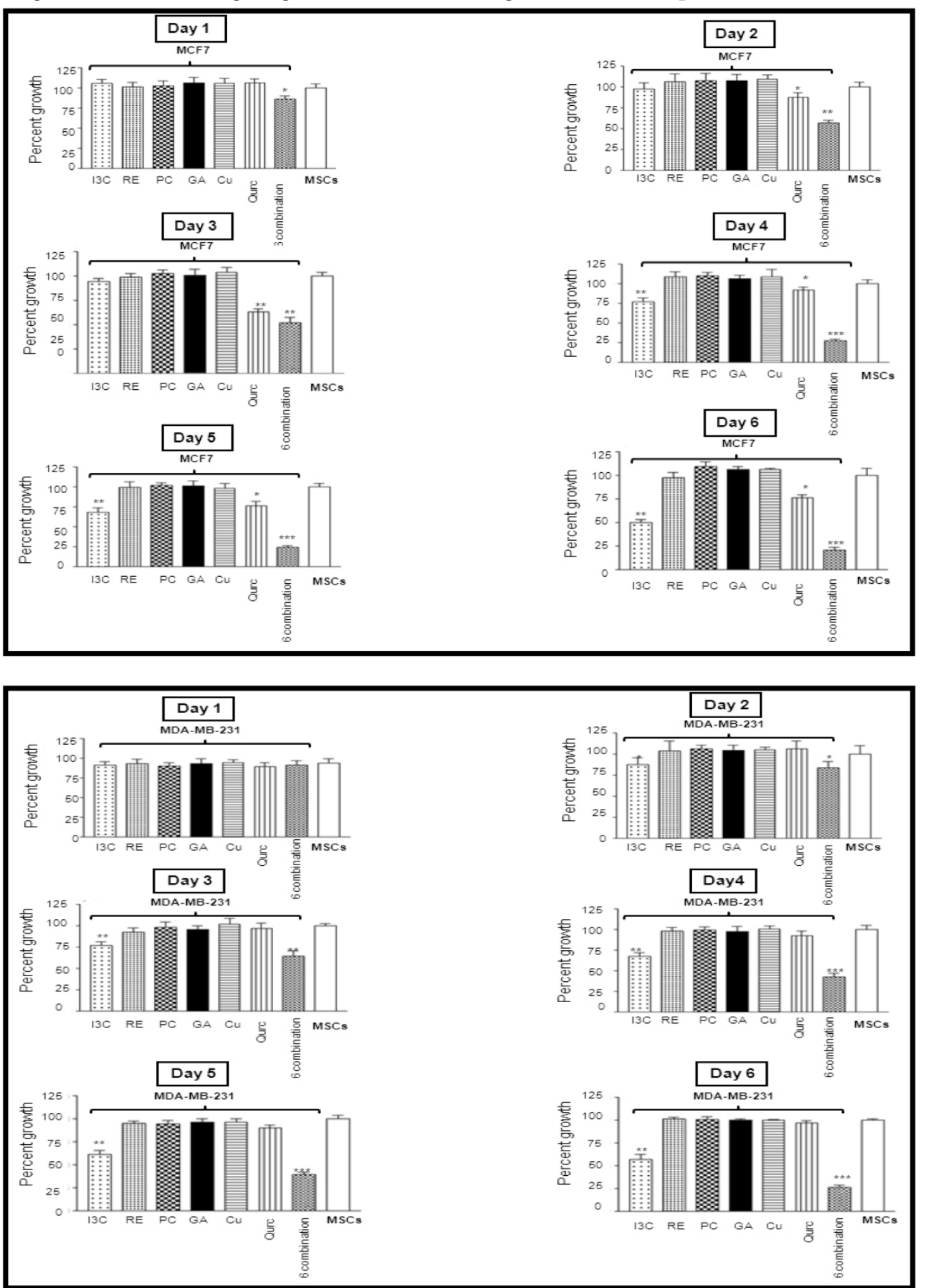

Fig I. Effects of each of the phytochemicals alone or their combination on cell proliferation of MCF7 cells (Top panel) and the highly invasive MDA-MB-23I cell line (Bottom panel) assayed with Alamar-Blue dye. Cells were treated over a 6 day period and Alamar-Blue assay was performed daily as described under methods. The data is expressed as percent of growth \pm SEM, as compared to the negative control MSCs (I00\%), unless noted otherwise. Level of significance is denoted as follows: *, $\mathrm{p}<0.05$; **, $\mathrm{p}<0.01$; ***, $\mathrm{p}<0.00 \mathrm{I}$. ferent pathways important for inhibiting cancer cell migration, invasion, proliferation, and survival. 


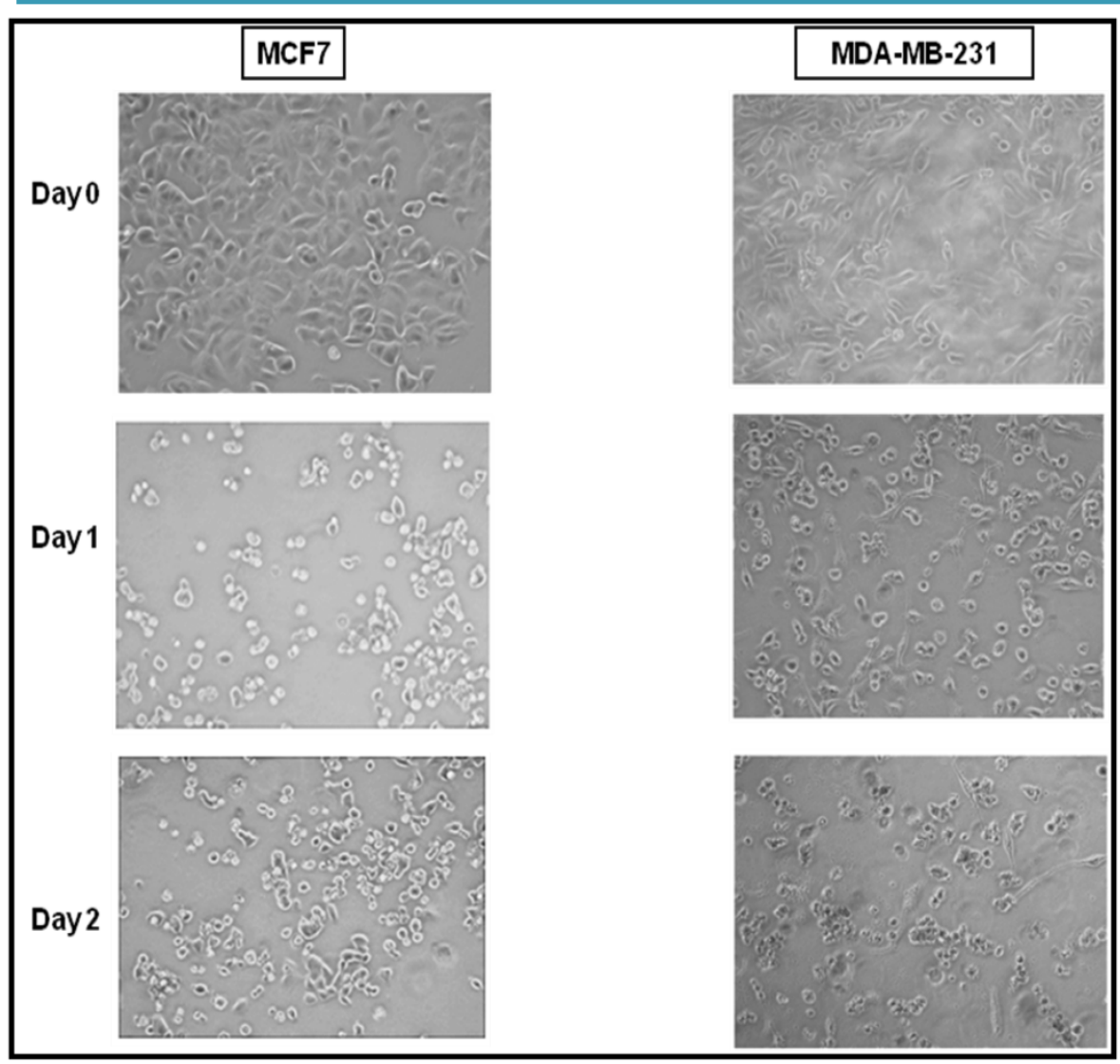

Fig 2. Effect of the phytochemical combination on MCF7 and MDA-MB-23I cell morphology. MCF7 and MDA-MB-23I cells at day 0 exhibited a smooth epithelial cell pattern with prominent nuclei. In contrast the cells treated with the 6-combination start to lose cell-cell contact and attain more rounded shape at day I. By day 2 , cells cluster together, demonstrate membrane blebbing, and start to detach from the dish (original magnification, $\mathrm{X} 100$ ).

Fig 3. Flow cytometry data analysis of MCF7 and MDA-MB-23I cells after the 6-combination treatment. Data demonstrate a non-significant effect of phytochemicals at cell cycle stages after 6h of 6-combination treatment of both cell lines. Meanwhile, a significant increase in cell apoptosis at $24 \mathrm{~h}$ of MCF7 cells treatment with six phytochemicals in combination, and only a small increase in cell apoptosis of treatment of MDA-MB-23I cells. In the top panel, representative flow cytometric diagrams are given. $\mathbf{A}$ : MDA-MB-23I control at $0 \mathrm{hr}$. B: MDA-MB-23I treated for 24 hrs. C: MCF-7 control. D: MCF-7 treated for $24 \mathrm{hrs}$.
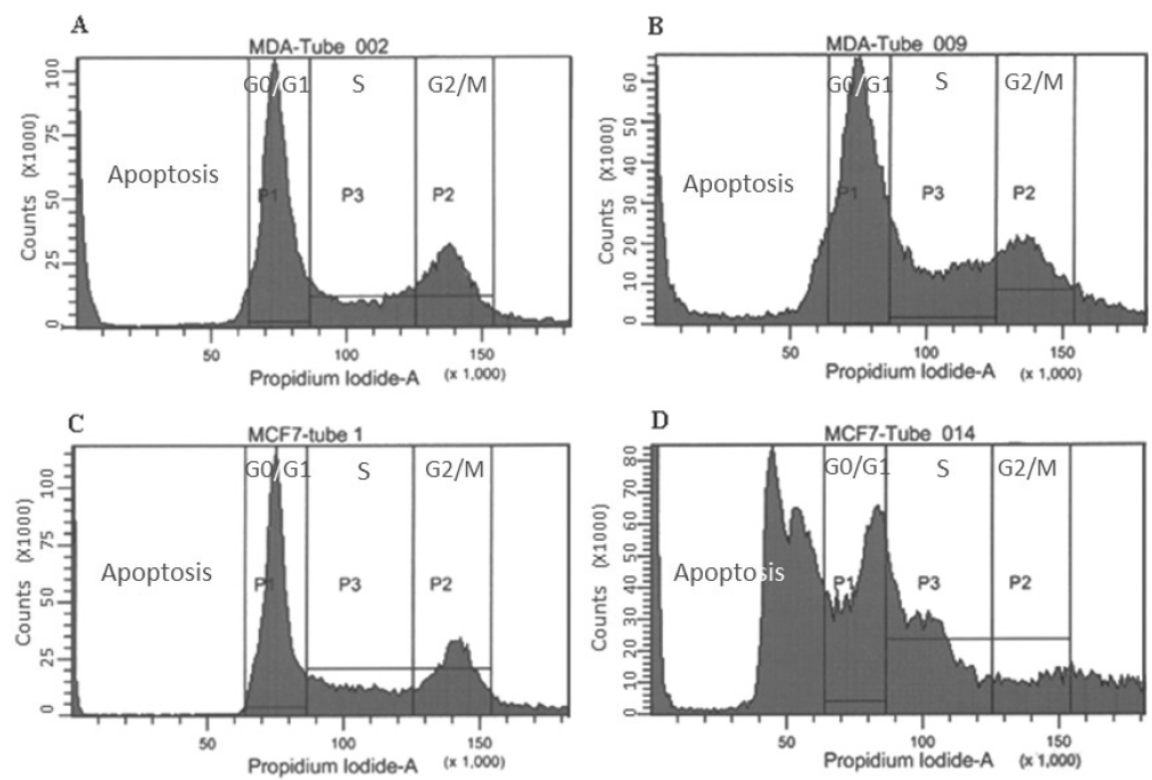

\begin{tabular}{|c|c|c|c|c|c|c|c|c|}
\hline \multirow{2}{*}{ 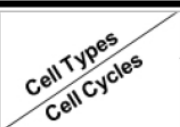 } & \multicolumn{4}{|c|}{$\overline{M C F 7}$} & \multicolumn{4}{|c|}{ MDA-MB-231 } \\
\hline & $\mathrm{Oh}$ & $6 \mathrm{~h}$ & $12 \mathrm{~h}$ & $24 \mathrm{~h}$ & Oh & $6 \mathrm{~h}$ & $12 \mathrm{~h}$ & $24 \mathrm{~h}$ \\
\hline G0/G1\% & 47.7 & 55 & 46.1 & 27.9 & 58.2 & 60.3 & 62 & 57.2 \\
\hline $\mathbf{S} \%$ & 17.1 & 19.4 & 21.3 & 6.74 & 14.9 & 19.4 & 20.8 & 18.3 \\
\hline G2/M $\%$ & 27.5 & 19.8 & 8.29 & 1.58 & 22.5 & 15.5 & 12 & 16.4 \\
\hline Apoptosis $\%$ & 0.17 & 0.59 & 20.3 & 45.6 & 1.53 & 2.46 & 3.29 & 4.78 \\
\hline
\end{tabular}




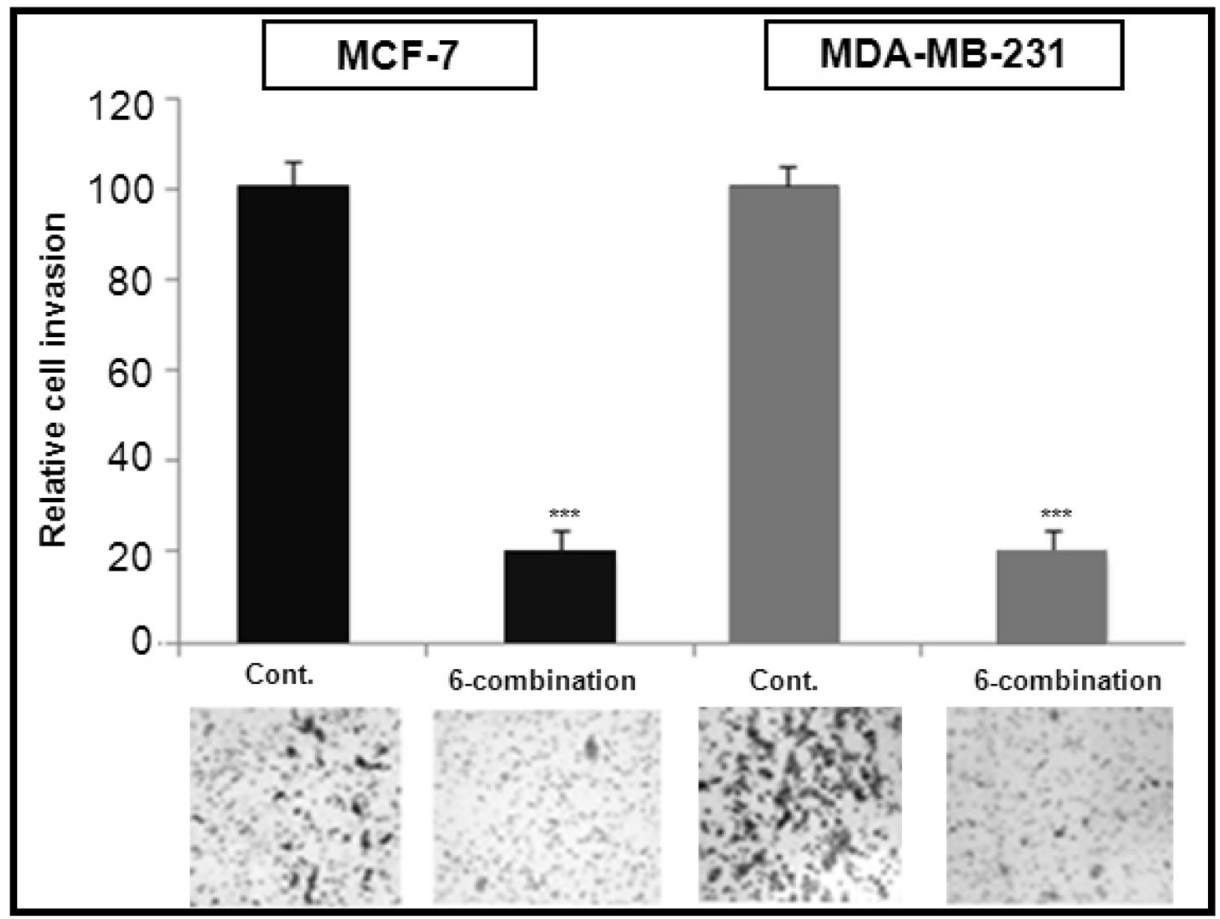

Fig 4. Determination of the phytochemical effect on the invasiveness of MCF7 and MDA-MB-23I cell lines. Cell invasiveness is demonstrated by Boyden chamber invasion assay. Images of Boyden chamber membranes (bottom panels) represent the number of invaded cells, illustrating the difference in invaded cell numbers. Note the reduction in invasion capability of MCF7 and MDA-MB-23I cell after 6-combination treatment. All data were performed in triplicate and in three independent experiments. Black bars represent MCF7 cells invasion, while the grey bars represent MDA-MB-23I cell invasion (student's two-tailed t-test, $* \mathrm{P}<0.05$; $* * \mathrm{P}<0.0 \mathrm{I}$, $* * * \mathrm{P}<0.00 \mathrm{I}$ ).

\section{Determination of cellular targets of 6-combination treatment}

In the microarray analysis, a time dependent down-regulation of the anti-apoptotic Bcl-2 and SVV genes were recognized. Additionally, significant down regulation of CD44 and mutant p53 (cancer cell migration promoting proteins) were observed, and a less significant down regulation of cell cycle regulatory proteins CDK4 and $\mathrm{Rb}$ were detected (Table 1$)$. Further, microarray analysis revealed 4 genes that were highly up-regulated by the SC treatment: ARC [activity-regulated cytoskeleton associated protein, 13.5 fold]; GADD45B [growth arrest and DNA damage-inducible, beta, 18.5 fold]; MYLIP [myosin regulated light chain interacting protein, 18.3 fold); CDNK1C (cyclin dependent kinase inhibitor 1C(p57, Kip2), 16.9fold]. These represent genes involved in regulation of cell motility, apoptosis, cell cycle and survival.

\section{DISCUSSION}

Dietary phytochemicals have been shown to be effective in killing a variety of cancer cells in vitro, when used in pharmacologic concentrations [1-30]. In spite of these effective anti-cancer actions, the use of these phytochemicals at high concentrations not achievable by oral dosing after long regimen of treatment [35] have prevented their use in clinical studies. Here we evaluated the separate and combined effects of six phytochemicals (Curcumin, Genistein, I3C, C-PC, - RE and Quercetin) at their bioavailable levels against primary and metastatic BC cell lines over a period of 6 days. We hypothesized that these compounds would work together in an additive / synergistic manner by altering the expression of several cellular targets to alter several tumor hallmarks such as uncontrolled cell growth and proliferation, evasion of cell apoptotic signals, induction of cell motility and invasion, and to inhibit cancer progression.

Table I. Microarray data of the 6-combination targeted genes, showing a significant down-regulation at mRNA levels of anti-apoptotic proteins $\mathrm{Bcl}-2$ and SVV, cancer cell migratory protein CD44, mutant $\mathrm{p} 53$, cell cycle regulatory proteins CDK4 and $\mathrm{Rb}$. (negative values refer to fold change of down-regulated genes, the positive values refer to the fold change of up-regulated genes after the 6-combination treatment).

\begin{tabular}{llll}
\hline Genes & $6 \mathrm{hr}$ & $12 \mathrm{hr}$ & $24 \mathrm{hr}$ \\
\hline CDK4 & 0.62 & -0.01416 & -1.3 \\
Bcl-2 & -1.21018 & -3.30757 & -3.44386 \\
$\mathrm{Rb}$ & 0.23648 & 0.14952 & -0.64276 \\
mutant Tp53 & 0.68784 & 0.022082 & -2.12756 \\
CD44 & -0.62 & -1.86 & -2.4 \\
SVV & -0.42 & -0.5 & -1.6 \\
\hline
\end{tabular}



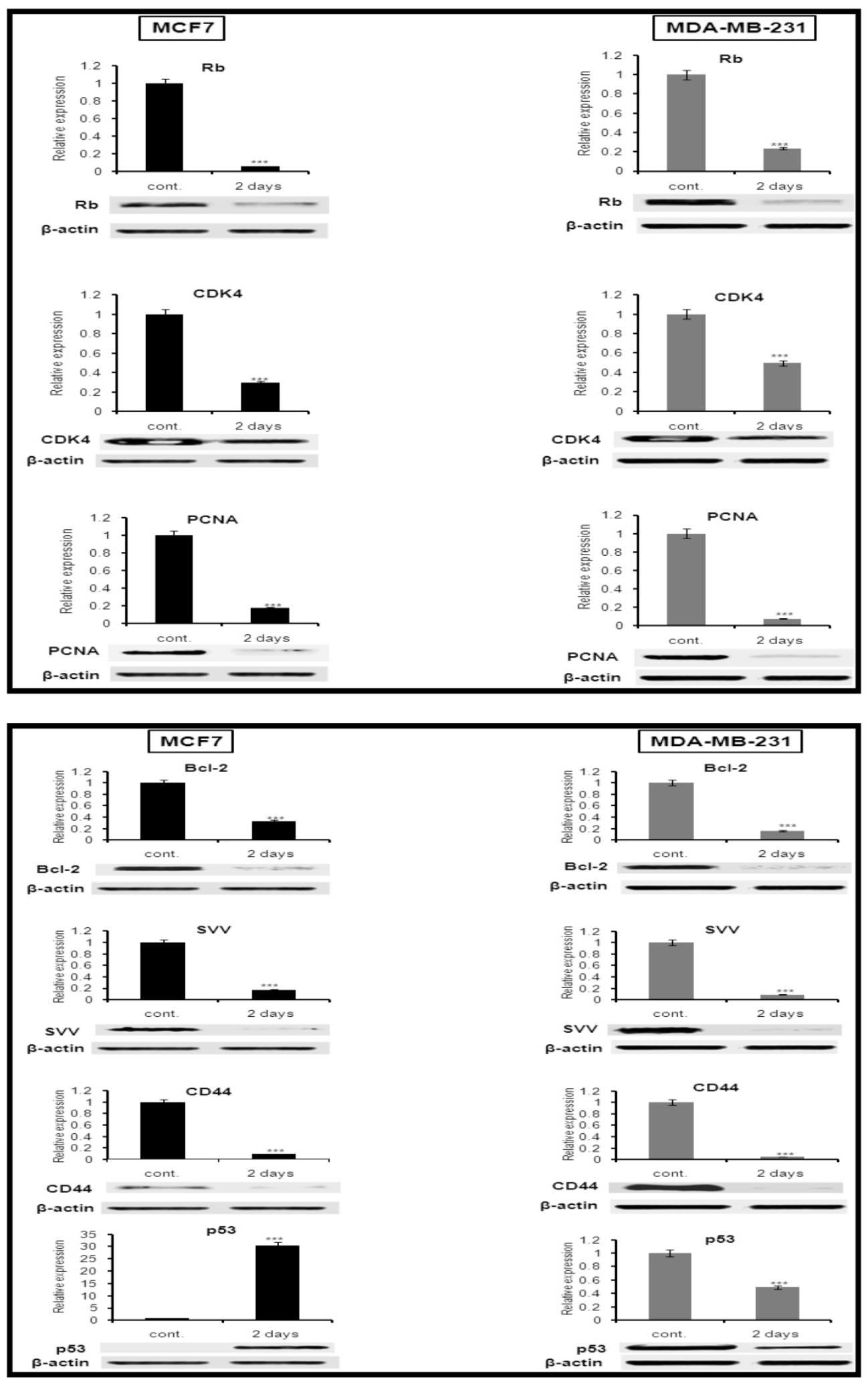

Fig 5. Molecular mechanisms of the 6-combination inhibitory actions on cell migration, invasion and induction of cell apoptosis in MCF-7 and MDA-MB-23 I cell lines Cells were treated with the 6-combination for $48 \mathrm{hrs}$, protein lysates were collected and examined by western blot analysis as described under methods. All bands were quantified and normalized against $\beta$-Actin that was used as loading Control. Top panel: Synergistic down-regulation of cell proliferation marker PCNA and cell cycle regulators Rb, CDK4. Bottom panel: Down regulation of anti-apoptotic BcL-2, SVV and the cell metastatic marker cell adhesion molecule CD44 (marker of cell metastasis and BC stem cell marker) in both cell lines after 48hr from cell treatment with the 6 phytochemicals combination. Down-regulation of the MDA-MB-23I mutant P53 and up-regulation of P53 wild type in MCF7 was interestingly analyzed. 
The separate and combined effect of these phytochemicals on the proliferation of primary MCF7 BC cells and the highly metastatic MDA-MB-231 BC cells were tested through the application of the above mentioned phytochemicals in cell growth media. Our results revealed that exposure of MCF7 cells to the 6-combination resulted in a significant reduction in proliferation rate by day 2 of the experiment, an inhibition that increased significantly by the sixth day resulting in a nearly $80 \%$ reduction in cell growth. Interestingly, no reduction in proliferation rate of the control MSCs was seen with the six phytochemicals combination treatment (Figure 1). Similarly, the combined phytochemicals also inhibited the proliferation of the highly metastatic MDA-MB-231 cells by day three of treatment, which like the MCF7 cells decreased by more than $80 \%$ on the sixth day of the experiment (Figure 1). The resistance that MDA-MB-231 cells showed to phytochemical treatment is consistent with the known resistance of metastatic cancer cells to chemo-and radio-therapeutic treatment. These results, which demonstrate that the effect of the combination treatment is much greater than the effect of the individual phytochemicals, supports our hypothesis that the combined phytochemicals are working together to inhibit cell growth and migration (metastasis).

In order to investigate whether the reduction in cellular proliferation induced by 6-combination treatment results from inhibition of the cell cycle or by a reduction in number of cells due to induced cell death, we assessed the changes in cell morphology after day 1 and day 2 of 6 -combination treatment. The data revealed significant changes in cell morphology reflecting the hallmarks of cell apoptosis by day 1 of treatment. At day 2 dead cells showed up floating in the culture media of both cell lines. Further consistent with an increase in apoptosis, FACS analysis showed a significant increase in apoptosis of MCF7 cell line after $12 \mathrm{hr}$ of SC treatment, reaching highly significant levels $(\sim 70 \%)$ at $24 \mathrm{hrs}$ (Figure 3$)$. FACS data indicated only 5\% apoptosis for MDA-MB-231 after $24 \mathrm{~h}$ of SC treatment (Figure 3), which as described above, may result from the increased resistance of these metastatic $\mathrm{BC}$ cells to therapy during this short period of treatment (Figure1, bottom panel).

In order to understand the molecular mechanisms contributing to the observed decrease in proliferation and increased apoptosis, we examined the expression levels of several proteins known to be important for these biological processes (Figure 5). We observed a significant reduction in expression of PCNA in both cell lines. Further, we observed a significant decrease in the expression of the cell cycle regulators CDK4 and its downstream target $\mathrm{Rb}$, indicating a possible arrest of the cell cycle (Figure 5, top panel). Moreover, there was a significant inhibition in the expression levels of the two anti-apoptotic proteins Bcl-2 and SVV, a member of the inhibitor of apoptosis protein (IAP) family, after 6-combination treatment of both cell lines (Figure 5 bottom panel).

The 6-combination also had a profound effect on the migration and invasive capacity of both MCF7 and MDA-MB-231 cells. Treated cells were unable to migrate into the wounded area in a wound healing assay during a time period in which the control cells healed properly. Similarly SC treatment caused an $80 \%$ reduction in the invasive capability of both cell lines. CD44 is a molecule that has been shown to be responsible for cancer cell motility and for invasion, through the expression of its downstream targets cortactin (CTN) [33] and survivin, as shown by us previously [36]. Here, we demonstrated that CD44 levels were decreased upon treatment with the 6-combination (Figure 5). This decrease in CD44 expression suggests that the 6-combination affects cancer cell motility and invasiveness, in part through altering its expression.

We found the results of the expression of p53 highly interesting. . The expression of wild type p53 (Wt p53) is altered in cancer cells by either down-regulation of its expression as in most primary cancer cells, or by mutation of its DNA-binding domain as in most metastatic tumors (37-39). All of these changes suppress p53 function as a guard of the human genome during cell replication. Consistent with this fact, the presence of mutated p53 in colon cancer patients is often used as an indicator of poor survival and its level is greatly increased in high graded metastatic cases acting as adverse prognostic factor for cancer treatment [40]. Further, mutated p53 is known to have an oncogenic function by inducing cell growth, enhancing colony formation, and promoting invasion and migration [41]. Inhibition of mutated p53 in HepG2, hepatocellular carcinoma cell lines using interfering RNA technology results in inhibition of cell growth and invasion ability [42]. In contrast to the oncogenic nature of mutated p53, wt p53 is known to have tumor suppressor functions [39].

In the data presented here, a combination of 6 phytochemicals induced a marked reduction in the expression of mutated p53 in MDA-MB-231 metastatic cell line. Loss of the mutated or oncogenic form of p53 in MDA-MB-231 cells and an increase in expression of Wt p53, the tumor suppressor form of p53 in MCF7 cells is consistent with our observed alterations of the normal cancer phenotypes. The knock down of $\mathrm{Wt}$ P53 in MCF7 cells was previously demonstrated to increase the cell resistance for chemotherapy [43]. Interestingly, treatment of MCF7 cells with the six phytochemical combination greatly restored the ex- 
pression of $\mathrm{Wt} p 53$. This induction of $\mathrm{Wt} p 53$ would sensitize the primary $\mathrm{BC}$ cells for chemotherapy by suppressing the transcription of breast cancer resistance protein (BCRP) via NF-KB pathway [44]. The expression of $\mathrm{Wt}$ p53 and mutated p53 are regulated from the same promoter in both MCF7 and MDA-MB-231 cells. However, we observed very different effects on p53 expression between these two cell lines upon treatment with the 6-combination. Therefore, we believe that the effect of combination on the expression of wild type and mutant p53 can not necessarily occur at the level of transcription and is most likely caused by a post-transcriptional modification through regulation of hyper-methylation or histone de-acetylation enzymes.

Finally, we tested the change in expression levels of the above mentioned gene targets in the $\mathrm{BC}$ metastatic cell line MDA-MB-231 using microarray approach. The data showed significant down regulation of Bcl-2, SVV, CD44, mutant p53, CDK4 and Rb on the transcriptional level after $24 \mathrm{~h}$ of 6-combination treatment (Table 1). Further, new targets have been identified for the up-regulated genes at $24 \mathrm{~h}$ of 6-combination treatment. Interestingly, a recent in-vivo study of chemo-preventive effect has demonstrated the synergism of a phytochemical combination either through topical or dietary administration in preventing skin cancer development [45].

In summary, our data suggest that treatment of primary and highly metastatic BC cell lines with the physiologically relevant levels of six phytochemicals in combination causes a significant reduction in cell proliferation, motility, invasion with a concomitant induction of apoptosis. Six combination treatment caused a marked suppression in proliferation, motility and invasion of even the resistant MDA-MB-231 cells. Moreover, the study indicated that the phytochemical combination markedly inhibited the expression of the cell adhesion molecule CD44, which is metastasis-initiating factor. CD44 is also known as a marker for BC stem cells, the only sub population of cancer cells which have the ability to promote new tumor formation at secondary sites and are known to have a high resistance for cancer chemo-and radio therapies. In the present study we did not investigate the effects of this phytochemical cocktail on BRCA1 and BRCA2. However previous studies [46-48] have shown that BRCA1 and BRCA2 are molecular targets for four of the six compounds (Indole-3-carbinol, Resveratrol, Genistein and Curcumin) used in this phytochemical cocktail. Thus, it will be of great interest to evaluate any synergistic or additive effects of this cocktail on expression of BRCA1 and BRCA2. Further, we previously demonstrated that I3C and RE synergize to effectively kill ovarian cancer cells [27], thus making this super cocktail effective against this cancer also. Future experiments include animal studies using mouse xenograft model to evaluate the in-vivo toxicity and efficacy of the phytochemical super cocktail treatment to prevent and/or regress BC tumors as well as possible use of the highly up-regulated novel genes as markers to follow-up progress of therapy.

\section{ABBREVIATIONS}

BC: Breast Cancer; MCF-7, MDA-MB-231: Breast cancer cell lines; SC: Super Combination MMP-1: Matrix metalloprotease; I3C: Indole-3-Carbinol ; ER: Estrogen receptor; RE: Resveratrol PR: Progesterone receptor; SC: Super Combination; ROS: Reactive oxygen species; MSC: Mesenchymal stem cells; DIM: 3'-dindolylmethane; PCNA: Proliferating cell nuclear antigen; PARP: Poly (ADP) ribose polymerase; $\mathrm{Rb}$ : Retinoblastoma protein; C-PC, PC: C-phycocyanin; CDK4, CDK6: Cyclin dependent kinases; DMB: Dibutyl nitrosamine; Bcl-2, Bcl- $\mathrm{X}_{\mathrm{L}}$ : Antiapoptotic protein; GA: Genistein; SVV: Survivin; CUR: Curcumin; Qurc: Quercetin; PBS: Phosphate-buffered saline; CSC: Cancer stem cells; BSA: Bovine serum albumin; MDR: Multi-drug resistance; FBS: Fetal Bovine Serum; Bax, Bak: pro-apoptotic proteins; NF-kB: Nuclear factor $k B$.

\section{ACKNOWLEDGEMENTS}

The authors wish to acknowledge research funding support as well as a fellowship to Mohamed Abdraboh from Egyptian ministry of Education. This study was partially supported by the Eminent Scholar XXXVIII Professorship award to Shubha K. Ireland. The assistance of Dr. Udai Pandey with the submission of this manuscript is also gratefully appreciated.

\section{COMPETING INTERESTS}

Dr. Madhwa HG Raj: A patent is being submitted for a phytochemical cocktail to maintain breast health.

Dr. Shailaja G Raj: Based on the findings in this research, a nutritional supplement for breast health (Breast Healthguard Formula) is being prepared for commercialization by Protegene Corporation.

All other authors declare that no competing interest exists.

\section{REFERENCES}

1. Subramaniam D, Ramalingam S, Houchen CW, Anant S. Cancer stem cells. A novel paradigm for cancer prevention and treatment. Mini Rev Med Chem 2010; 103:59-71.

2. Kawasaki BT, Hurt EM, Mistree T, Farrar WL. Targeting cancer stem cells with phytochemicals. Mol Interv 2008; 8:174-84.

3. Gullett NP, Ruhul Amin AR, Bayraktar S, et al. Cancer prevention with natural compounds. Semin Oncol 2010; 37:258-81.

4. Moiseeva EP, Manson MM. Dietary chemopreventive phytochemicals: Too little or too much? Cancer Prev Res (Phila) 2009; 2:611-6. 
5. Mehta RG, Murillo G, Naithani R, Peng X. Cancer chemoprevention by natural products: How far have we come? Pharm Res 2010; 27:950-61.

6. Barnes S. Effect of genistein on in vitro and in vivo models of cancer. J Nutr 1995; 125:777S-83S.

7. Zhou JR, Gugger ET, Tanaka T, Guo Y, Blackburn GL, Clinton SK. Soybean phytochemicals inhibit the growth of transplantable human prostate carcinoma and tumor angiogenesis in mice. J Nutr 1999; 129:1628-35.

8. Moiseeva EP, Almeida GM, Jones GD, Manson MM. Extended treatment with physiologic concentrations of dietary phytochemicals results in altered gene expression, reduced growth, and apoptosis of cancer cells. Mol Cancer Ther 2007; 6:3071-9.

9. Rowe DL, Ozbay T, O'Regan RM, Nahta R. Modulation of the BRCA1 protein and induction of apoptosis in triple negative breast cancer cell lines by the polyphenolic compound curcumin. Breast Cancer 2009; 3:61-75.

10. Yodkeeree S, Ampasavate C, Sung B, Aggarwal BB, Limtrakul P. Demethoxycurcumin suppresses migration and invasion of MDA-MB-231 human breast cancer cell line. Eur J Pharmacol 2010; 627:8-15.

11. Hua WF, Fu YS, Liao YJ, et al. Curcumin induces down-regulation of EZH2 expression through the MAPK pathway in MDA-MB-435 human breast cancer cells. Eur J Pharmacol 2010; 637:16-21.

12. Liu $\mathrm{Q}$, Loo WT, Sze SC, Tong $\mathrm{Y}$. Curcumin inhibits cell proliferation of MDA-MB-231 and BT-483 breast cancer cells mediated by down-regulation of NFkappaB, cyclinD and MMP-1 transcription. Phytomedicine 2009; 16:916-22.

13. Kurahashi N, Iwasaki M, Sasazuki S, et al. Soy product and isoflavone consumption in relation to prostate cancer in japanese men. Cancer Epidemiol Biomarkers Prev 2007; 16:538-45.

14. Matsuo K, Hiraki A, Ito $\mathrm{H}$, et al. Soy consumption reduces the risk of non-small-cell lung cancers with epidermal growth factor receptor mutations among japanese. Cancer Sci 2008; 99:1202-8.

15. Suzuki T, Matsuo K, Tsunoda N, et al. Effect of soybean on breast cancer according to receptor status: A case-control study in japan. Int J Cancer 2008; 123:1674-80

16. $\mathrm{Li} \mathrm{Y,} \mathrm{Ellis} \mathrm{KL,} \mathrm{Ali} \mathrm{S,} \mathrm{et} \mathrm{al.} \mathrm{Apoptosis-inducing} \mathrm{effect} \mathrm{of} \mathrm{chemotherapeutic}$ agents is potentiated by soy isoflavone genistein, a natural inhibitor of NF-kappaB in BxPC-3 pancreatic cancer cell line. Pancreas 2004; 28:e90-5.

17. Raffoul JJ, Wang Y, Kucuk O, Forman JD, Sarkar FH, Hillman GG. Genistein inhibits radiation-induced activation of NF-kappaB in prostate cancer cells promoting apoptosis and G2/M cell cycle arrest. BMC Cancer 2006; 6:107.

18. Tonetti DA, Zhang Y, Zhao H, Lim SB, Constantinou AI. The effect of the phytoestrogens genistein, daidzein, and equol on the growth of tamoxifen-resistant T47D/PKC alpha. Nutr Cancer 2007; 58:222-9.

19. Acharya A, Das I, Singh S, Saha T. Chemopreventive properties of indole-3-carbinol, diindolylmethane and other constituents of cardamom against carcinogenesis. Recent Pat Food Nutr Agric 2010; 2:166-77.

20. Nguyen HH, Lavrenov SN, Sundar SN, et al. 1-benzyl-indole-3-carbinol is a novel indole-3-carbinol derivative with significantly enhanced potency of anti-proliferative and anti-estrogenic properties in human breast cancer cells. Chem Biol Interact 2010; 186:255-66.

21. Rahman KM, Sarkar FH, Banerjee S, et al. Therapeutic intervention of experimental breast cancer bone metastasis by indole-3-carbinol in SCID-human mouse model. Mol Cancer Ther 2006; 5:2747-56.

22. Chen T, Wong YS, Zheng W. Induction of G1 cell cycle arrest and mitochondria-mediated apoptosis in MCF-7 human breast carcinoma cells by selenium-enriched spirulina extract. Biomed Pharmacother 2009; Epub.

23. Ismail MF, Ali DA, Fernando A, Abdraboh ME, Gaur RL, Ibrahim WM, Raj $\mathrm{MH}$, Ouhtit A. Chemoprevention of rat liver toxicity and carcinogenesis by spirulina. Int J Biol Sci 2009; 5:377-87.

24. Hayashibara T, Yamada Y, Nakayama S, et al. Resveratrol induces downregulation in survivin expression and apoptosis in HTLV-1-infected cell lines: A prospective agent for adult $\mathrm{T}$ cell leukemia chemotherapy. Nutr Cancer 2002; 44:193-201.

25. Aggarwal BB, Bhardwaj A, Aggarwal RS, Seeram NP, Shishodia S, Takada Y. Role of resveratrol in prevention and therapy of cancer: Preclinical and clinical studies. Anticancer Res 2004; 24:2783-840.

26. Harikumar KB, Kunnumakkara AB, Sethi G, et al. Resveratrol, a multitargeted agent, can enhance antitumor activity of gemcitabine in vitro and in orthotopic mouse model of human pancreatic cancer. Int J Cancer 2010; 127:257-68.

27. Raj MH, Abd Elmageed ZY, Zhou J, et al. Synergistic action of dietary phyto-antioxidants on survival and proliferation of ovarian cancer cells. Gynecol Oncol 2008; 110:432-8.

28. Murakami A, Ashida H, Terao J. Multitargeted cancer prevention by quercetin Cancer Lett 2008. 269:315-25.

29. Duo J, Ying GG, Wang GW, Zhang L. Quercetin inhibits human breast cancer cell proliferation and induces apoptosis via Bcl-2 and Bax regulation. Mol Med Rep. 2012; 5:1453-6.

30. Zhang $\mathrm{H}$, Zhang $\mathrm{M}, \mathrm{Yu} \mathrm{L}$, Zhao $\mathrm{Y}, \mathrm{He} \mathrm{N}$, Yang $\mathrm{X}$. Antitumor activities of quercetin and quercetin-5',8-disulfonate in human colon and breast cancer cell lines. Food Chem Toxicol. 2012; 50:1589-99

31. Nebe B, Peters A, Duske K, Richter DU, Briese V. Influence of phytoestrogens on the proliferation and expression of adhesion receptors in human mammary epithelial cells in vitro. Eur J Cancer Prev 2006; 15:405-15.

32. Kim JH, Lee BK, Lee KW, Lee HJ. Resveratrol counteracts gallic acid-induced down-regulation of gap-junction intercellular communication. J Nutr Biochem 2009; 20:149-54.
33. Hill A, McFarlane S, Mulligan $\mathrm{K}$, Gillespie $\mathrm{H}$, Draffin JE, Trimble A, Ouhtit A Johnston PG, Harkin DP, McCormick D, Waugh DJ. Cortactin underpins CD44-promoted invasion and adhesion of breast cancer cells to bone marrow endothelial cells. Oncogene 2006; 25:6079-91.

34. Leung TH, Ching YP, Yam JW, Wong CM, Yau TO, Jin DY, Ng IO. Deleted in liver cancer 2 (DLC2) suppresses cell transformation by means of inhibition of RhoA activity. Proc Natl Acad Sci U S A 2005; 102:15207-12.

35. Scott EN, Gescher AJ, Steward WP, Brown K. Development of dietary phytochemical chemopreventive agents: Biomarkers and choice of dose for early clinical trials. Cancer Prev Res (Phila) 2009; 2:525-30.

36. Abdraboh ME, Gaur RL, Hollenbach AD, Sandquist D, Raj MH, Ouhtit A. Survivin is a novel target of CD44-promoted breast tumor invasion. Am J Pathol 2011, 179:555-63.

37. Ebina M, Martinez A, Birrer MJ, Ilona Linnoila R. In situ detection of unexpected patterns of mutant p53 gene expression in non-small cell lung cancers. Oncogene 2001; 20:2579-86.

38. Gasco M, Shami S, Crook T. The p53 pathway in breast cancer. Breast Cancer Res 2002;4:70-6.

39. Oren M, Rotter V. Mutant p53 gain-of-function in cancer. Cold Spring Harb Perspect Biol 2010; 2:a001107.

40. Riddle ND, Gorden L, Rojiani MV, Hakam A, Rojiani AM. CD44 and p53 immunoexpression patterns in NF1 neoplasms - indicators of malignancy and infiltration. Int J Clin Exp Pathol 2010; 3:515-21.

41. Goh AM, Coffill CR, Lane DP. The role of mutant $\mathrm{p} 53$ in human cancer. J Pathol 2011 Jan;223(2):116-26

42. Xie Z, Chng WJ, Tay KG, Liu SC, Zhou J, Chen CS. Therapeutic potential of antisense oligodeoxynucleotides in downregulating p53 oncogenic mutations in cancers. Biotechnol Lett 2010. 33:221-8

43. Nadkarni A, Rajesh P, Ruch RJ, Pittman DL. Cisplatin resistance conferred by the RAD51D (E233G) genetic variant is dependent upon p53 status in human breast carcinoma cell lines. Mol Carcinog 2009; 48:586-91.

44. Wang $\mathrm{X}, \mathrm{Wu} \mathrm{X}$, Wang $\mathrm{C}$, et al. Transcriptional suppression of breast cancer resistance protein (BCRP) by wild-type p53 through the NF-kappaB pathway in MCF-7 cells. FEBS Lett 2010;584:3392-7.

45. Kowalczyk MC, Kowalczyk P, Tolstykh O, Hanausek M, Walaszek Z, Slaga TJ. Synergistic effects of combined phytochemicals and skin cancer prevention in SENCAR mice. Cancer Prev Res (Phila) 2010;3:170-8.

46. Fan S, Meng Q, Auborn K, Carter T, Rosen EM. BRCA1 and BRCA2 as molecular targets for phytochemicals indole-3-carbinol and genistein in breast and prostate cancer cells. Br J Cancer 2006;94:407-26

47. Chirnomas D, Taniguchi T, de la Vega M, Vaidya AP, Vasserman M, Hartman AR, Kennedy R, Foster R, Mahoney J, Seiden MV, D'Andrea AD. Chemosensitization to cisplatin by inhibitors of the Fanconi anemia/BRCA pathway. Mol Cancr Ther 2006;5:952-61

48. Papoutsis AJ, Lamore SD, Wondrak GT, Selmin OI, Romagnolo DF. Resveratrol prevents epigenetic silencing of BRCA1 by the aromatic hydrocarbon receptor in human breast cancer cells. J Nutr 2010;140:1607-14

\section{AUTHOR BIOGRAPHY}

Madhwa HG Raj: (M.Sc., Ph.D) Dr. Raj is Professor in department of Obstetrics-Gynecology and Member of Stanley S Scott Cancer Center at Louisiana State University Health Sciences Center, New Orleans, Louisiana. He has published more than 70 publications on reproductive endocrinology, male contraception and cancer. He was an invited participant in the Indo-US Science and Technology program of $\mathrm{NIH}$, for male contraceptive vaccine development and was participating laboratory sponsor for Rockefeller Foundation Technology Transfer Program. He has served as reviewer for many journals including Endocrinology, International Journal of Cancer, Breast Cancer Research and Treatment, Breast Cancer Research, American Journal of Obstetrics and Gynecology, Science, Respirology, Fertility and Sterility, Life Sciences and Molecular and Cellular Biochemistry. He is the originator of the concept of rendering phytochemicals effective at bioavailable levels by inclusion in a super cocktail. He is currently developing a clinical trial program for use of phytochemical super-cocktail in breast cancer chemoprevention/ 
chemotherapy, in collaboration with Protegene Corporation. He can be contacted through his e-mail address: rajmadhwa@yahoo.com

Allal Ouhtit: (Ph.D) He is currently Associate Professor in Department of Genetics at Sultan Quboos University School of Medicine, Muscat, Oman. He has more than 25 publications on various aspects of cancer.

Rajiv lochan Gaur: (Ph.D) He is currently working as Research Associate at Department of Pathology, Stanford University, Palo Alto, CA. His expertise includes molecular mechanism of diseases, mutation analysis, diagnostics development and phytochemical action on cancers.

Mohamed Abdraboh: (Ph.D) He is currently faculty in Biology at University of Mansora, Egypt.

Shubha K. Ireland: (Ph.D) She is currently Professor of Biology at Xavier University of Louisiana. A recent former Chair, she is actively involved in externally funded studies on cancer research and regulation of secondary metabolism as well as in development and assessment of competency-based curricular reforms funded by the Howard Hughes Medical Institute.

Prakash N Rao: (PhD, MBA, FACHE, HCLD) He has served on the faculty of the LSU Health Sciences Center, New Orleans, and the Stanley Scott Cancer Center and later as Professor, Dept. of Surgery, and Director of the Transplant Evaluation Laboratory at the University of South Alabama Medical Center. Currently, Dr. Rao is the VP of Diagnostic and Research Operations at the New Jersey Organ and Tissue Sharing Network.

Andrew Hollenbach: (Ph.D) Dr. Hollenbach is currently Associate Professor, Department of Genetics at LSU Health Sciences Center, New Orleans.

Shailaja G Raj: (MD, FACOG, REI) She has served as Associate Professor and Acting Chief, Division of Reproductive Endocrinology, Department of Ob-Gyn, at Louisiana State University Health Sciences Center, School of Medicine in New Orleans. She developed and taught a course on all aspects of Breast, to medical students, Residents and Fellows. She has over 25 publications, is currently in private practice of Gynecology, Reproductive Endocrinology and Infertility. She is President of Protegene Corporation, which is commercializing a phytochemical super cocktail based on the findings in this research, as nutritional supplement for breast health (Breast Health-guard formula) in women. She can be contacted through her e-mail address: Gshailaja.raj@gmail.com. 